\title{
Melioidosis manifesting as severe emaciation and clinically indolent liver abscesses, in a child with Beta thalassemia major
}

\author{
*M B K C Dayasiri ${ }^{1}$, R M Mudiyanse ${ }^{1}$, H D W S Kudagammana ${ }^{2}$, M I Rifaya ${ }^{3}$, P Dissanayaka ${ }^{2}$, C \\ Jeyaratnasingam ${ }^{3}$, U Nawaratna ${ }^{3}$
}

Sri Lanka Journal of Child Health, 2016; 45(2):130-133

DOI: http://dx.doi.org/10.4038/sljch.v45i2.7617

(Key words: Melioidosis; emaciation; liver abscess; beta thalassaemia major)

\section{Introduction}

Melioidosis is a pyogenic infection caused by the soilassociated bacterium Burkholderia pseudomallei ${ }^{1}$. It is an important cause of community-acquired sepsis in Southeast Asia and northern Australia ${ }^{1,2}$. Melioidosis is an emerging infection in India, with a reported prevalence of $7 \%^{3}$. Sri Lanka, positioned between 5 10 degrees north, is situated in the endemic belt and has similarities in weather and environmental conditions with other countries in South Asia. However, Sri Lanka has been considered non-endemic for melioidosis ${ }^{4}$. This disease being commoner in rural populations where it goes undetected and the high mortality before a diagnosis is made, may have contributed to the so far perceived low prevalence of melioidosis in Sri Lanka ${ }^{5}$.

It is classically characterized by pneumonia and multiple abscesses, with a mortality rate of up to $40 \%{ }^{6}$. However, little is currently known about bacterial pathogen evolution and adaptation within the host during acute infection ${ }^{7}$. Infection is acquired by inoculation or inhalation and is more common in patients with underlying chronic disease. Melioidosis has a myriad of clinical manifestations and can present either as acute fulminant infection or chronic indolent debilitating illness. However, it has also been reported in asymptomatic afebrile patients ${ }^{8}$. Melioidosis in

${ }^{1}$ University Paediatrics Unit, Teaching Hospital, Peradeniya, Sri Lanka, ${ }^{2}$ Department of Microbiology, Teaching Hospital Peradeniya, Sri Lanka, ${ }^{3}$ General Hospital Ampara, Sri Lanka

*Correspondence: mbkcdayasiri@gmail.com

(Received on 13 November 2014: Accepted after revision on 19 December 2014)

The authors declare that there are no conflicts of interest

Personal funding was used for this project.

Open Access Article published under the Creative Commons Attribution CC-BY cc (†) neonates is also recognised with possible fetomaternal transmission ${ }^{9}$. In this case report we present a 7 year old child from Ampara with Beta thalassemia major who had chronic melioidosis manifesting as severe emaciation and multiple liver abscesses.

Case report

A 7 year old child with Beta thalassaemia major, on regular monthly blood transfusions since the age of five months, presented with a history of poor weight gain and progressive emaciation over the past 7 months. He was transferred from Ampara General Hospital to Teaching Hospital Peradeniya for further evaluation. His parents and younger sister were carrying the thalassemia trait and his elder sister was getting regular blood transfusions for congenital dyserythropoietic anaemia. His serum ferritin level was $4,720 \mu \mathrm{g} / \mathrm{L}$ on admission and recent evaluations showed no evidence of diabetes, hypothyroidism, hypoparathyroidism or cardiac haemosiderosis. However, there had been a recent increase in the size of liver $(7 \mathrm{~cm}$ below right costal margin with normally lying upper border) associated with progressive abdominal distension and generalised wasting of the body (Figure 1).

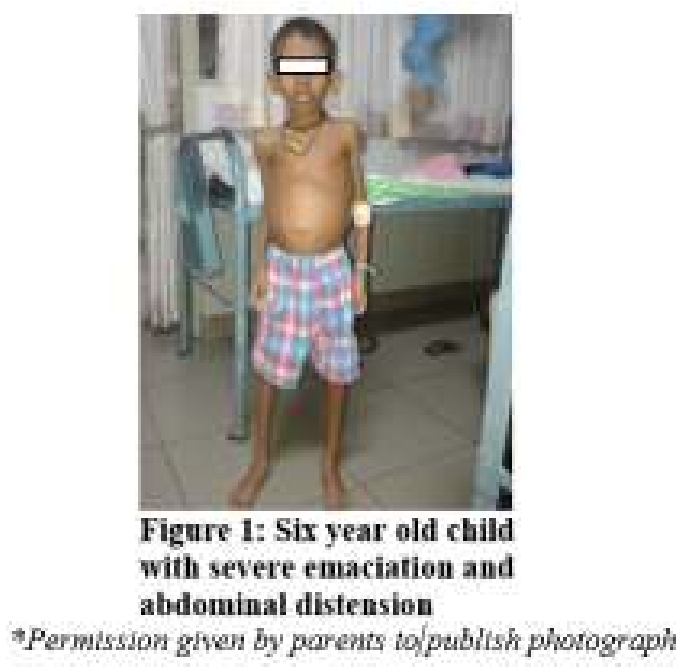


During the same period child had been otherwise asymptomatic apart from a febrile illness a month before which was treated with intravenous antibiotics as for sepsis. There was no history of abdominal pain, icterus, altered stool / urine colour or any bleeding manifestations. There was no history recurrent infections, chronic diarrhoea or recent increase in requirement for blood transfusions. Child had a poor socioeconomic background and diet quality and quantity had been poor. Physical examination found an afebrile, emaciated child with pallor and hepatosplenomegaly without icterus or lymphadenopathy.

ESR serial values were above $110 \mathrm{~mm}$ in the $1^{\text {st }}$ hour with elevated C-reactive protein (CRP) $(>96 \mathrm{mg} / \mathrm{L})$. Mantoux test and sputum for acid fast bacilli were negative. Hepatitis B surface antigen and hepatitis C antibody levels were negative. Blood culture showed no growth and blood gases were normal. Human immunodeficiency virus antibodies were not detected. Chest x-ray did not reveal lymphadenopathy, malignancy or evidence of lung abscess. Ultrasound scan (USS) of abdomen showed multiple focal lesions within liver with hepatomegaly and was suggestive of resolving abscesses or malignancy. The kidneys, pancreas, adrenal glands and spleen were normal apart from splenomegaly. Blood picture was not suggestive of a haematological malignancy and full blood count showed normal cell count and differential count. Serum alpha feto protein was $1.1 \mathrm{IU} / \mathrm{ml}$ and was also not supportive of liver malignancy. Liver profile showed normal liver transaminase (SGOT/ SGPT) values, normal gamma glutamyl transferase, normal bilirubin (direct and indirect fractions) with marginally reduced total protein and albumin levels. Computed tomography of the abdomen was suggestive of multiple liver abscesses though malignancy could not be excluded.

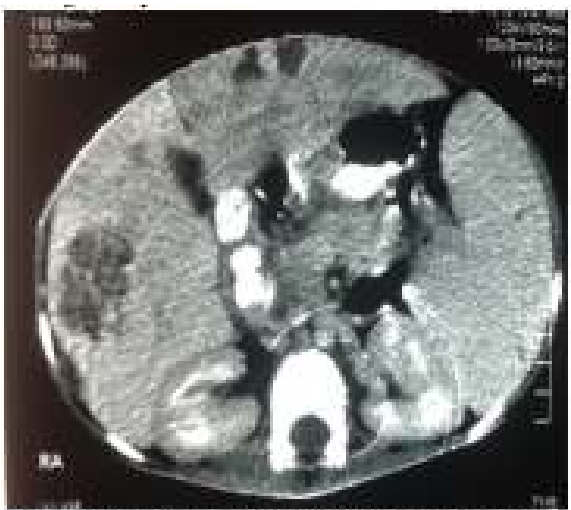

Figure 2: Computed tomography showing muttiple tiver abscesses
Subsequently child underwent USS guided liver biopsy and histology showed extensive hepatocellular necrosis, fibrosis and chronic hepatitis in addition to haemosiderosis. Melioidosis antibody levels were arranged as radio imaging was suggestive of poorly resolving chronic infection of the liver. Following admission child was given intravenous (IV) antibiotics including meropenem and vancomycin to cover possible poorly resolving chronic infection. During ward stay he developed rapid onset septic arthritis of the right elbow joint and it was planned to do arthrotomy and culture of the joint fluid. While child was awaiting the orthopaedic intervention, melioidosis cultures and antibody titres became positive. Subsequently long term treatment with IV antibiotics was arranged with intravenous meropenem for one month (Phase 1) and oral cotrimoxazole for 6 months (Phase 2). Child was redirected to the local paediatrician for continuation of further care.

\section{Discussion}

The first published report of melioidosis in Sri Lanka was in 1927 in a European tea broker resident in Sri Lanka and it is also believed to be first reported in South Asia ${ }^{10}$. There had been no reported cases of melioidosis up to $1994^{7}$. Corea et al. (2012) stated there had been seven reported cases of melioidosis in Sri Lanka by year 2008 and the numbers will further increase with improved detection and diagnosis. All seven cases reported were aged above 25 years and their comorbidities included diabetes mellitus, renal disease and alcoholism which were likely to be predisposing factors.

In the current case report the child was from a rural area of the country and concurrent beta thalassemia major had been a predisposing comorbidity in acquisition of melioidosis. Child did not become acute ill with a fulminant course though he had developed severe emaciation and clinically indolent multiple liver abscesses. Thalassemia has been shown to be associated with melioidosis and it has also been reported in older children who underwent splenectomy following hypersplenism from South East Asia ${ }^{11}$. The fact that our child is from a rural area reinforces the previously published information that the disease may be underreported in less resourceful areas?

As the clinical presentation of melioidosis varies, diagnosis on clinical grounds alone is not easy. Therefore, definitive diagnosis is based on isolation of the organism from the affected sites and serological evidence. Samples obtained from sites of infection like 
sputum, abscess aspirations, joint fluid aspirations, wound or rectal swabs, urine and blood etc. can be cultured. The organism grows easily on commonly using bacteriological culture media but identification is difficult due to unfamiliarity of laboratory staff about the unusually variable colony morphology and inconstant performance of initial biochemical tests. Therefor the final confirmation requires the use of molecular based PCR or MALDI-TOF in a reference laboratory.

An indirect haemagglutination test (IHA), various enzyme-linked immunosorbent assays (ELISAs), and other serologic assays are available to detect specific antibody production against the pathogen. In endemic areas, their usefulness is limited by high rates of background antibody positivity. In acute septicaemic melioidosis, IHA and ELISA are often initially negative, but repeat testing may show seroconversion. Thus, paired samples with seroconversion, rising titres or extremely high titres will support the diagnosis of melioidosis.

Blood culture obtained from the child on $22^{\text {nd }}$ August 2014 grew a mixed growth of two organisms and one isolate gave similar performance that is suggestive of $B$ pseudomallei, with safety pin appearance in the Gram stain and was pink on Mac Conky agar on 48 hours with positive oxidase test though primary subculture antibiogram was not typical. His serology by IHA was very high giving confirmation of the diagnosis with a titre of $1 / 10240$.

Principal choice of antibiotics used in acute infection (first phase) comprises a cephalosporin, usually ceftazidime, or a carbapenem, usually meropenem. Alternatives such as cefepime and imipenem have both been proposed for acute therapy, and imipenem has been successfully used in clinical practice ${ }^{12}$. Duration of treatment of acute infection is currently debated however; recently updated therapeutic guidelines ${ }^{13}$ recommend a minimum duration of two weeks that needs to be extended when deep seated infections are present. Cotrimoxazole and folic acid can be used as adjunct therapy with extended infections involving nervous system, bones, and joints. Cotrimoxazole, doxycycline and coamoxyclav are recommended for eradication (phase 2) of Melioidosis and minimum duration of treatment is three months ${ }^{14}$.

\section{Acknowledgments}

The authors acknowledge Dr. Enoka Corea, Consultant Microbiologist and Senior Lecturer in
Microbiology, for the valuable contribution in evaluating antibody titres using Indirect Haemagglutination Test at Department of Microbiology, Faculty of Medicine, University of Colombo.

\section{References}

1. Dance DA. Melioidosis: tip of the iceberg. Clinical Microbiology Reviews 1991; 4:5260.

PMid: 2004347 PMCid: PMC358178

2. White NJ. Melioidosis. Lancet 2003; 361(9370): 1715-22. http://dx.doi.org/10.1016/S01406736(03)13 374-0

3. Kang G, Rajan DP, Ramakrishna BS, Auken HM, Dance DA. Melioidosis in India. Lancet 1996; 347:1565-6. http://dx.doi.org/10.1016/S01406736(96)90 723-0

4. Cheng AC, Currie BI. Melioidosis: Epidemiology, pathophysiology and management. Clinical Microbiology Reviews 2005; 18: 383-416. http://dx.doi.org/10.1128/CMR.18.2.383416.2005

PMid: 15831829 PMCid: PMC1082802

5. E Corea, V Thevanesam, S Perera, I Jayasinghe, A Ekanayake, J Masakorala, et al. Melioidosis in Sri Lanka: an emerging infection. Sri Lanka Journal of Infectious Diseases 2012; 1 (2):2-8. http://dx.doi.org/10.4038/sljid.v2i1.3801

6. Wiersinga WJ, Currie BJ, Peacock SJ. New England Journal of Medicine 2012; 367: 1035-44. http://dx.doi.org/10.1056/NEJMra1204699 PMid: 22970946

7. Price EP, Hornstra HM, Limmathurotsakul $\mathrm{D}$, et al. Within-host evolution of Burkholderia pseudomallei in four cases of acute melioidosis. PLoS Pathog 2010; 6: e1000725-e1000725. 
http://dx.doi.org/10.1371/journal.ppat.10007

25

PMid: 20090837 PMCid: PMC2799673

8. Green R, Mankikar DS. Afebrile cases of Melioidodis. British Medical Journal 1949; 1(4598): 308-11. http://dx.doi.org/10.1136/bmj.1.4598.308

PMid: 18123518 PMCid: PMC2049401

9. Noyal M, Harish BN, Bhat V, Parija SC. Neonatal Melioidosis: A case report from India. Indian Journal of Medical Microbiology 2009; 27:260-3. http://dx.doi.org/10.4103/0255-0857.53213

PMid: 19584512

10. Denny CR, Nicholls L. Melioidosis in a European. Ceylon Journal of Science 1927; 2:37-40.

11. TS Hoc, CT Deng, R Khuzaiah. Melioidosis in a splenectomised boy with beta Thalassemia Major. Southeast Asian Journal of Tropical Medicine and Public Health 1993; 24(3): 601-2.

PMid: 8160075

12. Ulett, G.C.; Hirst, R.; Bowden, B.; Powell, K.; Norton, R. A comparison of antibiotic regimens in the treatment of acute melioidosis in a mouse model. Journal of Antimicrobial Chemotherapy 2003; 51: 7781.

http://dx.doi.org/10.1093/jac/dkg011

PMid: 12493790
13. Cheng, A.C.; Currie, B.J. Melioidosis: epidemiology, pathophysiology, and management. Clinical Microbiology Reviews 2005; 18: 383-416. Erratum in: Clinical Microbiology Reviews 2007; 20: 533. http://dx.doi.org/10.1128/CMR.18.2.383416.2005

PMid: 15831829 PMCid: PMC1082802

14. Chaowagul $\mathrm{W}$ et al. Open-label randomized trial of oral trimethoprim-sulfamethoxazole, doxycycline, and chloramphenicol compared with trimethoprim- sulfamethoxazole and doxycycline for maintenance therapy of melioidosis. Antimicrobial Agents and Chemotherapy 2005; 49: 4020-5. http://dx.doi.org/10.1128/AAC.49.10.40204025.2005

PMid: 16189075 PMCid: PMC1251512 